\title{
Autoimmune Thyroid Disease
}

\author{
John E. Freitas, M.D.
}

Marked alterations in the level of circulating thyroid hormone frequently induce cutaneous changes. Such visible sequelae of hyperthyroidism or hypothyroidism can be among the first manifestations of thyroid dysfunction. The etiology of such thyroid dysfunction is frequently attributable to autoimmune thyroid disease, as manifested by Graves' disease (diffuse toxic goiter) or Hashimoto's thyroiditis (chronic lymphocytic thyroiditis). What is the primary immunologic disturbance that induces or facilitates autoimmune thyroid disease? Although many details remain to be elucidated, the hypothesis expounded by Volpè is attractive and appears consistent with the experimental and clinical data available. ${ }^{1}$ In his view, each autoimmune endocrine disorder is due to an organ-specific defect in suppressor $T$ lymphocyte function which allows the survival of a "forbidden" clone of self-reactive T lymphocytes. Having arisen by normal random mutation, the forbidden clone is then free to interact with its antigen, initiating a localized cell-mediated immune process. The same self-reactive T lymphocytes also function as "helper" lymphocytes, influencing the antibody-producing B lymphocytes to manufacture self-directed antibodies. As suggested by Volpè's hypothesis, both cell-mediated and humoral immunity appear to play a dominant role in the pathogenesis of Graves' disease and Hashimoto's thyroiditis. The specific defect and its sequelae in each disease process will be detailed further under separate headings. Although Graves' disease and Hashimoto's thyroiditis are best viewed as separate entities, both diseases coexist in some patients. The extent of such an association may be dependent upon that patient's specific defect in immune surveillance (suppressor T cell function) and the random appearance of the appropriate clone. Why both diseases affect women four times more frequently than men is unknown.

Address for reprints: John E. Freitas, M.D., Nuclear Medicine Department, William Beaumont Hospital, 3601 West 13 Mile Road, Royal Oak, MI 48072.

\begin{abstract}
From the Nuclear Medicine Department, William Beaumont Hospital, Royal Oak Michigan, The Department of Internal Medicine, University of Michigan Medical School, Ann Arbor, Michigan
\end{abstract}

\section{Graves' Disease}

This multisystem disorder is characterized by the presence of at least one of the following: (1) diffuse thyroid enlargement with hyperthyroidism, (2) infiltrative ophthalmopathy, or (3) infiltrative dermopathy. The latter two components are of importance, but space constraints prevent further descriptions here. An excellent review of endocrine ophthalmopathy has recently appeared. ${ }^{2}$

With the discovery of long-acting thyroid stimulator (LATS) in the serum of Graves' disease patients by Adams and Purvis in 1956, the disease became associated with autoimmune phenomenon. ${ }^{3}$ Subsequently, LATS, LATS protector, and human thyroid stimulator have been identified as immunoglobulins present in Graves' disease serum which stimulate the thyroid follicular cell. ${ }^{4-6}$ These various stimulators, collectively termed thyroid-stimulating immunoglobulins (TSI), are $\lg$ molecules acting as antibodies with thyroid-stimulating activity residing in the Fab fragment. ${ }^{7}$ TSI are almost invariably found in patients with active, untreated Graves' disease and appear to bind to the thyrotropin-stimulating hormone (TSH) receptor as their antigen. ${ }^{6.8}$ Other autoantibodies directed against either thyroglobulin or microsomal antigens can be identified in Graves' disease by sensitive assays. ${ }^{9}$ Such antibodies are not felt to play an important role in pathogenesis and appear to correlate directly with the degree of hyperthyroidism.

Organ-specific, cell-mediated immunity is also present in Graves' disease and Hashimoto's thyroiditis. In the direct leukocyte migration test, lymphocytes not sen- 
sitized to a particular antigen will migrate when exposed to that antigen. However, when previously sensitized lymphocytes are exposed to that antigen, migration inhibition factor (MIF) is produced and leukocyte migration decreases. Lymphocytes from patients with Graves' disease or Hashimoto's thyroiditis show marked inhibition of migration when exposed to particulate thyroid antigen, while normals do not. ${ }^{10}$ Similarly, Graves' disease lymphocytes produce TSI when exposed to phytohemagglutinin while normals do not. ${ }^{11}$ Thus, Graves' disease lymphocytes manifest both organspecific, cell-mediated and humoral immunity. Graves' disease appears to be a receptor-antibody disease in which autoantibodies stimulate the thyroid gland directly by their specificity for the TSH receptor. Graves' disease appears to be inherited as a polygenic tract, with TSI and Graves' disease present in 50 percent and 5 percent of siblings, respectively. Although increased frequency of HLA-B8 and Dw3 is seen in whites and Bw35 in Japanese with Graves' disease, this probably represents linkage dysequilibrium with a still yet unidentified gene.

\section{Diagnosis}

At any given time, a patient with Graves' disease may possess only one of its three major manifestations. In a particular patient, one or two of the manifestations may never appear; moreover, these three manifestations seem to occur and progress independently of each other in most patients. Thus, the patient's presentation varies with the manifestations present, their severity, the age of the patient, and any preexisting organ dysfunction. A diffuse goiter with hyperthyroidism is the most common manifestation of Graves' disease. The goiter typically has a spongy consistency with size varying from 30 to 120 gm estimated by palpation. Other signs of hyperthyroidism are lid lag and/or retraction, proptosis, stare, tremor, tachycardia, thyroid bruit, and velvety moist skin. When the majority of signs are present, an unmistakable picture results, but many patients with hyperthyroidism present with a paucity of signs. The major symptoms of hyperthyroidism are nervousness, heat in tolerance, fatigue, weight loss, increased perspiration, palpitations, and hyperdefecation. Nervousness predominates in younger patients, while cardiovascular symptoms are more often reported in the elderly.

Once the diagnosis of hyperthyroidism is considered, confirmation is usually achieved by measurement of serum thyroxine ( $\mathrm{T} 4$ ) concentration by radio immunoassay or competitive protein-binding analysis. ${ }^{12,13}$ However, serum T4 elevations are not pathognomonic of hyperthyroidism since alterations in the serum thyroxinebinding globulin (TBG) concentrations directly influence serum T4. To determine if elevated T4 values are due to hyperthyroidism or elevated TBG levels, estimation or direct measurement of the free (unbound) T4 value is necessary. In most situations, estimation of the free serum T4 concentration (free T4 index) suffices. The free $\mathrm{T} 4$ index ( $\mathrm{FTl}$ or $\mathrm{T} 7$ ) is the product of the total serum T4 value and the T3 resin uptake. The T3 resin uptake is inversely proportional to the number of unoccupied T4 binding sites available on TBG. In hyperthyroidism, the number of unoccupied binding sites is low and the T3 resin uptake high; while with TBG elevation, there is a surplus of unoccupied binding sites and the $\mathrm{T} 3$ resin uptake is low. Although free T4 concentration can be measured directly by equilibrium dialysis or radioimmunoassay, these techniques are not widely available and have not been shown to be superior to the FTI in clinical practice. ${ }^{14,15}$ Elevated FTI values are virtually diagnostic of hyperthyroidism in the appropriate clinical setting.

Most hyperthyroid patients demonstrate elevated serum T4 and T3 (triiodothyronine) concentrations. However, in occasional hyperthyroid patients, FTI values are normal, but T3 values are high. ${ }^{16}$ In the euthyroid subject, 80 percent of serum T3 is derived from peripheral deiodination of T4 rather than from thyroidal secretion; and T3 exerts the major effect of thyroid hormones on tissues. ${ }^{17}$ In hyperthyroidism, mean serum $T 4$ and $\mathrm{T} 3$ concentrations are two to three and three to four times the normal concentrations, respectively. ${ }^{18}$ Because of this disproportionate increase, some hyperthyroid patients demonstrate elevated T3 but normal FTI values (T3 thyrotoxicosis).

In some clinically hyperthyroid patients, equivocal elevations of FTI and T3 values cast doubt upon the diagnosis. To clarify the situation, tests assessing the integrity of the pituitary-thyroid axis, such as thyrotropinreleasing hormone (TRH) stimulation or T3 suppression, are required. Following TRH infusion, euthyroid subjects demonstrate a prompt rise in TSH, while hyperthyroid patients show no significant response (Fig. 1). Lack of TSH response to TRH is invariably seen in hyperthyroidism, but also occurs frequently in euthyroid Graves' disease and with autonomous thyroid adenoma. ${ }^{19}$ Because of simplicity and rapidity, the TRH stimulation test has largely supplanted the T3 suppression test. A useful scheme for investigation of suspected thyrotoxicosis is shown in Fig. 2.

\section{Treatment}

When Graves' disease with hyperthyroidism is confirmed, three therapeutic options are available: (1) antithyroid drugs, (2) surgery, and (3) radioiodine. All of these modalities can produce temporary or permanent cure of the disease. 
FiG. 1. TSH response to 500 ug of TRH given intravenously over 15 seconds. Euthyroid subjects show a TSH rise peaking at 30 minutes, while hyperthyroid patients show no significant TSH rise.

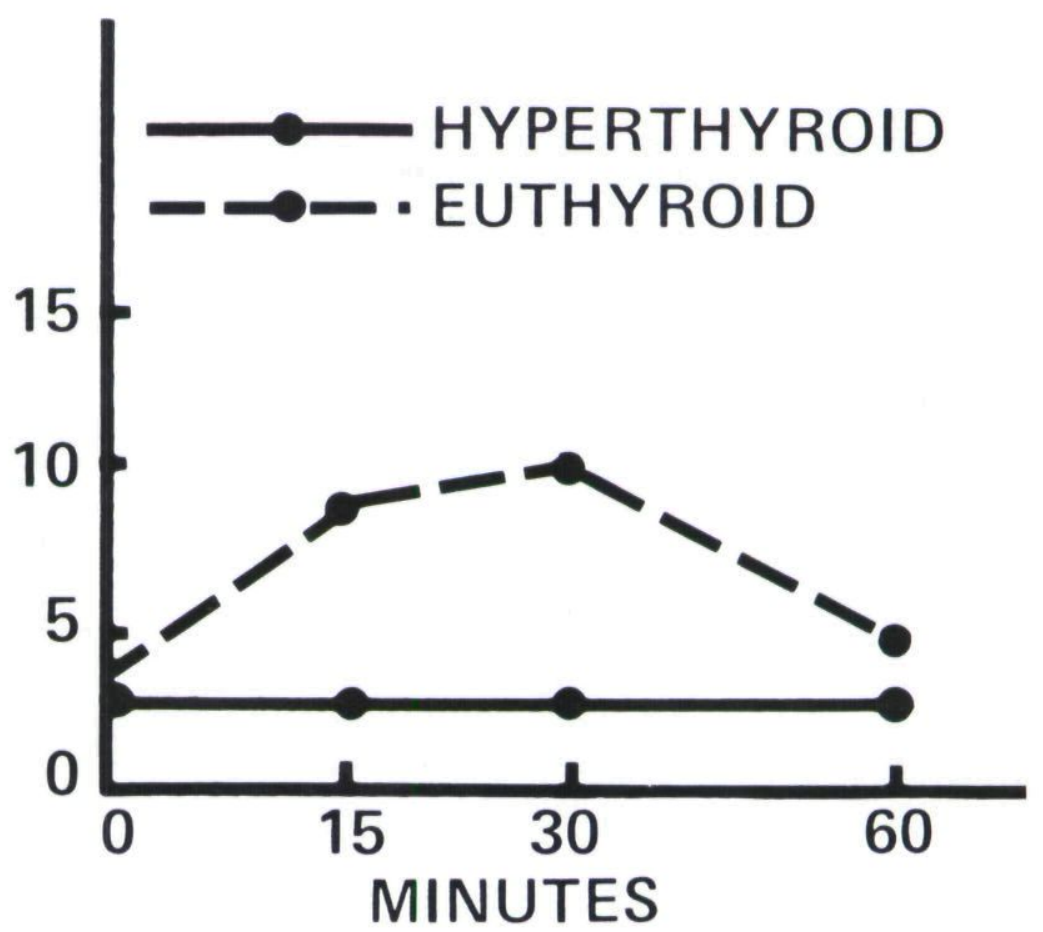

\section{Drug Therapy}

Drugs employed to treat hyperthyroidism either have a direct effect on the thyroid (thionamides, iodide, or lithium) or block the peripheral effect of thyroid hormone ( $\beta$-receptor blockers). The thionamides (propylthiouracil and methimazole) block thyroid hormone synthesis by inhibiting multiple steps in the T4, T3 pathway.
Such inhibition normalizes serum T4, T3 concentrations and depletes intrathyroid T4, T3 content. The amount of drug required to inhibit synthesis varies considerably and is determined in an individual patient by increasing the dose gradually until the desired effect is achieved. The thionamides probably do not influence the natural history of the disease, but control it for long intervals (months to years) waiting for a spontaneous remission.
FiG. 2. Diagnostic scheme for investigation of patient with suspected hyperthyroidism.

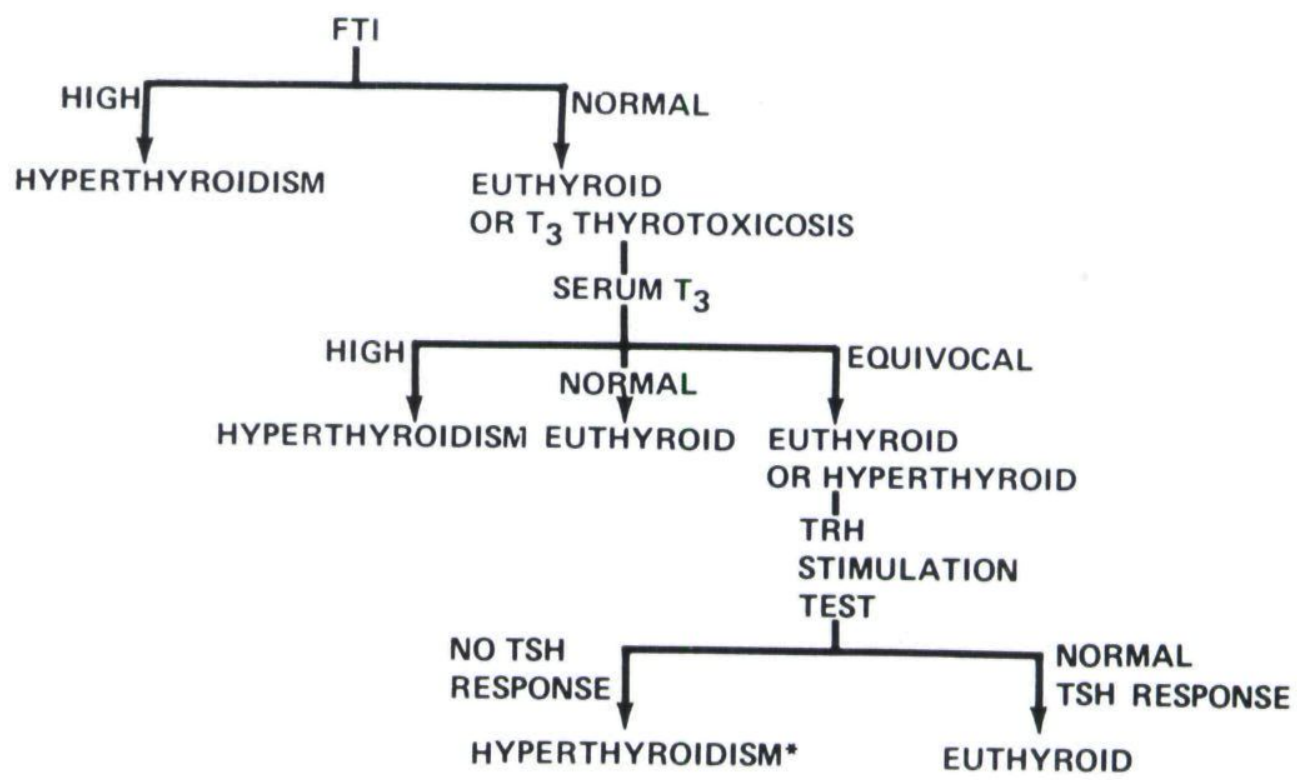

*EXCEPTIONS ARE

EUTHYROID GRAVES' DISEASE 
Unfortunately, current information demonstrates a progressive, unexplained fall in remission rate to less than 15 percent in unselected series. ${ }^{20}$ Other investigators have shown that persistence of thyroid-stimulating immunoglobulins or failure of decline in serum thyroglobulin during thionamide therapy are good predictors of relapse. ${ }^{21,22}$ Perhaps these indicators will prove useful in patient selection for long-term thionamide therapy. Both iodide and lithium impair thyroid hormone synthesis and block release of formed hormone. They have proven useful as supplemental agents to control hyperthyroidism, but are of little value when used alone as primary therapy.

$\beta$-receptor blocking agents, such as propranolol, reduce the $\beta$-adrenergic stimulated manifestations of hyperthyroidism probably through reduction of the synergistic effect of catecholamines and thyroid hormone upon the $\beta$-receptor. ${ }^{23}$ Propranolol also decreases the peripheral conversion of T4 to T3. However, recent work revealed no significant difference in symptomatic improvement between $\beta$-blockers that do or do not block T4 to T3 conversion. ${ }^{24}$ The major role of $\beta$-blockers is to provide symptomatic relief until thyrotoxicosis is relieved by definitive therapy. Bronchospasm and cardiac dysfunction are contraindications to propranolol use.

\section{Surgery}

Preoperative use of thionamides, iodide, and $\beta$-blockers has significantly reduced surgical morbidity and mortality, and subtotal thyroidectomy is the procedure of choice in selected patients. Unfortunately, as thyroid diagnosis and therapy have improved, the number of thyroid procedures and experienced thyroid surgeons has declined. As thyroidectomy experience declines, complications increase, as noted in a national survey review which revealed an overall mortality of 0.2 percent and the morbidity of 3 percent permanent hypoparathyroidism. ${ }^{25}$ The incidence of iatrogenic hypothyroidism is inversely proportional to the hyperthyroidism recurrence rate.

\section{Radioiodine}

Despite the recognized high incidence of iatrogenic hypothyroidism, I-131 is the most commonly used treatment modality because of its ease of administration, low cost, and lack of serious side effects. However, concern over possible long-term untoward effects, such as genetic damage, thyroid cancer, or leukemia, has restricted I-131's use in many centers. Fortunately, longterm follow-up studies have not identified such detrimental effects to date. ${ }^{26-28}$ Recently, the gonadal radiation dose following I-131 therapy has been shown to be no more than that realized from an IVP or barium enema. ${ }^{29}$

\section{Choice of Therapy}

The therapy selected by the patient and the treating physician are dependent upon many factors, such as patient age, concurrent disease, and individual preferences. I prefer radioiodine therapy for most hyperthyroid patients with the following exceptions. Radioiodine therapy is absolutely contraindicated during pregnancy, and such patients are best managed with the thionamides alone through pregnancy. After delivery, thionamides are stopped and radioiodine administered. Hyperthyroid patients with goiter-induced local compression symptoms should be treated surgically after adequate preoperative preparation.

Treatment of children with Graves' disease is a controversial issue. An initial trial of thionamide therapy of up to one year appears warranted in cooperative children, though remission rates of less than 30 percent are expected..$^{30}$ If spontaneous remission does not occur, I-131 therapy should be given, since it is safe and effective. ${ }^{26}$ Some thyroid surgeons advocate total thyroidectomy in children less than 10 years of age to decrease the recurrence rate. ${ }^{31,32}$ This must add to the morbidity rates in these children.

\section{Hashimoto's Thyroiditis}

This chronic destructive thyroiditis is characterized pathologically by diffuse lymphocytic infiltration, varying degrees of fibrosis, and follicular cell atrophy of the thyroid. Since Roitt first described the presence of circulating autoantibodies in patients with Hashimoto's thyroiditis, this disease has been accepted by many as an autoimmune disease. ${ }^{33}$ However, despite intensive investigation, our knowledge of its pathogenesis remains limited. Thyroidal antigens, such as thyroglobulin, are routinely released into the systemic circulation, yet this "exposure" of antigens does not normally induce thyroidal autoantibody synthesis. However, in patients with a defect in suppression $T$ cell function, as postulated by Volpè, these normal antigens sensitize a forbidden clone of T lymphocytes. ${ }^{34}$ These sensitized lymphocytes differentiate into (1) helper T cells which induce B lymphocyte production of autoantibodies, and (2) killer T cells which attack the thyroid directly. Thyroglobulin-antithyroglobulin complexes appear cytotoxic in vitro, and thyroid autoantibodies can apparently be cytotoxic in cooperation with killer T cells. ${ }^{35}$ Again, as in Graves' disease, the immune surveillance defect is a prerequisite for initiation of autoimmune thyroid disease. Such a defect appears inheritable as a polygenic tract with the presence of thyroid antibodies and overt thyroid disease in 50 percent and 25 percent, respectively, of siblings of involved patients. ${ }^{36-38}$ No association with specific histocompatibility antigens has been demonstrated.

Hashimoto's thyroiditis is the most common thyroid disease in the U.S., with an estimated prevalence 
of one to three percent of the population. The disease is usually detected by the physician's discovery of an asymptomatic small goiter which gradually enlarges if untreated. Hypothyroidism or occasionally, hyperthyroidism develop in the majority of patients with time.

\section{Diagnosis}

The presence of a goiter in a child or young adult strongly suggests Hashimoto's thyroiditis, which can be detected in more than 50 percent of such patients. Classically, the goiter is firm, with a bosselated surface, nontender, and less than $50 \mathrm{gm}$ in size. If the patient is clinically euthyroid or hypothyroid, a FTI, serum TSH, and thyroid antibodies are drawn initially. Since the disease presents no singly distinguishing feature, the use of multiple diagnostic criteria improves diagnostic accuracy (Table 1). ${ }^{39}$ If two or three criteria are present, the likelihood of Hashimoto's thyroiditis is greater than 65 percent and 85 percent respectively.

As thyroid destruction proceeds, serum TSH progressively increases to stimulate thyroidal T4, T3 production to maintain euthyroidism until hypothyroidism ensues. Thyroid antibodies in high titer are present in most patients with Hashimoto's thyroiditis. The assay procedure used most commonly is tanned red cell agglutination (TRCA) to detect either antithyroglobulin (ATG) or antimicrosomal antibodies (AM). ${ }^{40}$ Positive TRCA values
TABLE 1. Diagnostic Criteria for Hashimoto's Thyroiditis

\begin{tabular}{lc}
\hline \multicolumn{1}{c}{ Criteria } & Prevalence \\
\hline Firm, bosselated goiter & $90 \%$ \\
Presence of thyroid antibodies & $90 \% *$ \\
$\begin{array}{l}\text { Elevated serum TSH } \\
\text { Heterogeneous distribution of } \\
\text { tracer on thyroid scan }\end{array}$ & $30 \%^{*}$ \\
$\begin{array}{l}\text { Abnormal iodide-perchlorate } \\
\text { discharge test }\end{array}$ & $54 \% *$ \\
\hline
\end{tabular}

* Modified from reference 39

from ATG or AM antibodies are seen in approximately 55 percent and 75 percent, respectively (combined $90 \%)$, in histologically confirmed Hashimoto's thyroiditis. AM antibodies seem to correlate better with the extent of thyroiditis in the gland.

In patients in whom careful thyroid examination and in vitro studies have not sufficiently confirmed the diagnosis, a technetium pertechnetate thyroid scan should be obtained. Classically, the scan demonstrates enhanced thyroid uptake early with heterogeneous tracer distribution (Fig. 3). This pattern is not specific, but it is seen in greater than 50 percent with Hashimoto's thyroiditis. In occasional patients, further clarification is possible through either (1) the iodide-perchlorate test demonstrating a thyroidal organic-binding defect of
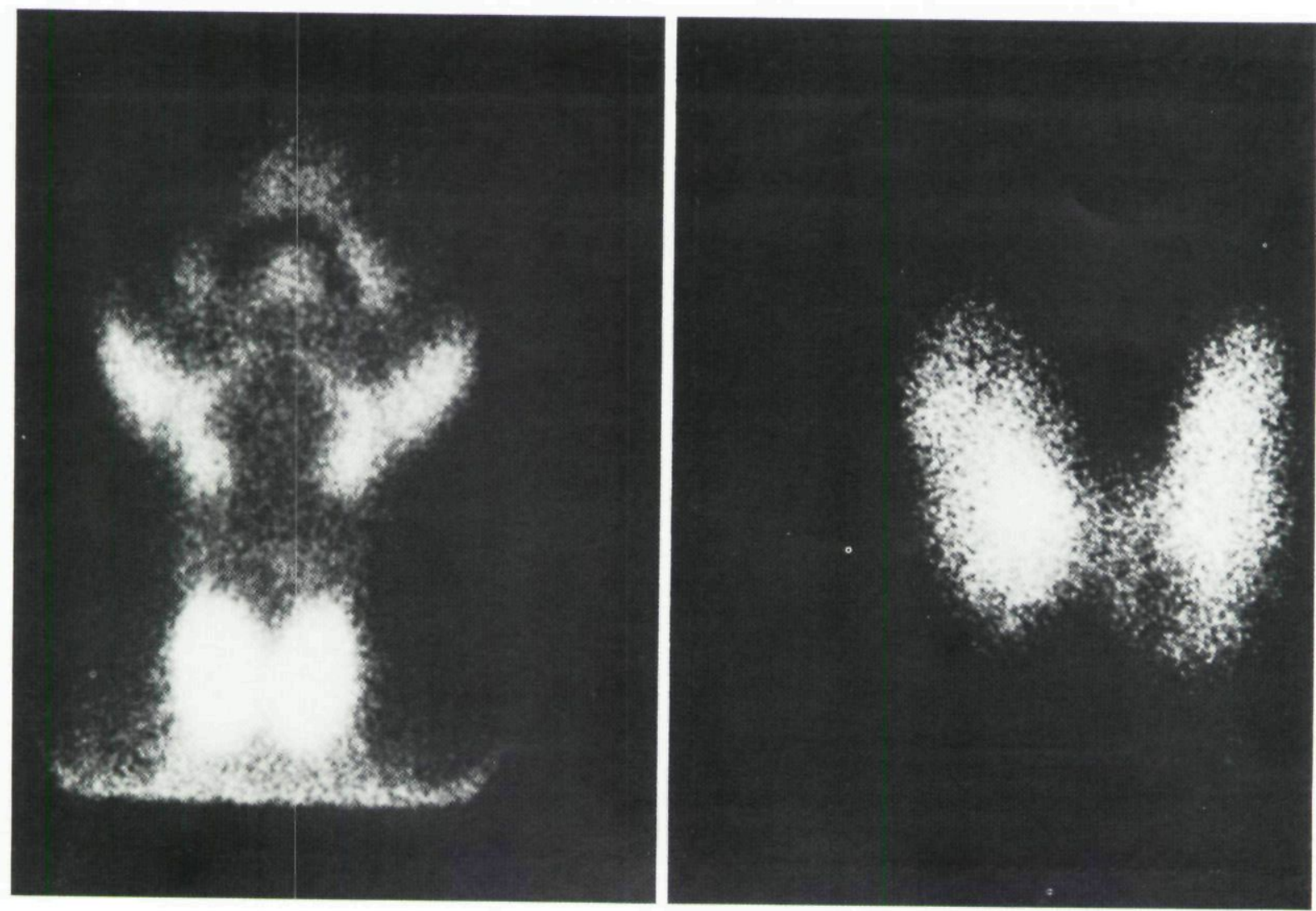

FIG. 3. A. One minute image (obtained five minutes after tracer injection) demonstrating increased thyroid trapping as compared with salivary glands. B. 100,000 count image obtained 20 minutes after tracer injection demonstrating heterogeneous tracer distribution throughout thyroid. 
iodine, a common finding in Hashimoto's thyroiditis, or (2) TRH stimulation test demonstrating a TSH hyperresponse indicative of decreased thyroidal reserve.

\section{Treatment}

In a euthyroid patient with elevated thyroidal antibodies, no therapy is instituted unless a goiter greater than $30 \mathrm{gm}$ is present. Levothyroxine $(0.15$ to $0.2 \mathrm{mg} /$ day) prevents further conspicuous enlargement and decreases goiter size in most. In patients with elevated serum TSH, but normal FTI, the majority develop clinically detectable hypothyroidism over a three-year follow-up, and should be treated when the elevated TSH is detected with levothyroxine as above..$^{41}$ Likewise, patients with elevated serum TSH and low serum FTI are hypothyroid, and levothyroxine therapy is required even if patient is asymptomatic.

\section{Conclusions}

The autoimmune thyroid diseases develop in a predisposed population with a defect in immune surveillance. This defect allows production of thyroid-directed autoantibodies that are either stimulatory to (TSI) or destructive of (antimicrosomal) the patient's thyroid gland. Proper diagnosis and therapy requires an understanding of the specific immunologic mechanisms involved, their sequelae, and corrective measures of the induced thyroid dysfunction.

\section{References}

1. Volpè R, Farid NR, Von Westrap C, et al: The pathogenesis of Graves' disease and Hashimoto's thyroiditis. Clin Endocrinol $3: 239,1974$

2. Gorman CA: The presentation and management of endocrine ophthalmopathy. Clin Endocrinol Metab 7:67, 1978

3. Adams DD, Purvis HD: Abnormal responses in the assay of thyrotropin. Proc Univ Otago Med Sch 34:11, 1956

4. Kriss JP, Pleshakov V, Chief JR: Isolation and identification of the long-acting thyroid stimulator and its relation to hyperthyroidism and circumscribed pretibial myxedema. J Clin Endocrinol Metab 24:1005, 1964

5. Adams DD, Kennedy TH: Evidence to suggest that LATS protector stimulates the human thyroid gland. J Clin Endocrinol Metab $33: 47,1971$

6. Mukhtar ED, Smith BR, Pyle GA, et al: Relation of thyroid stimulating immunoglobulin to thyroid function and effects of surgery, radioiodine and antithyroid drugs. Lancet i:713, 1975

7. Smith BR, Dorrington KJ, Munro DS: The thyroid stimulating properties of long-acting thyroid stimulator and $G$ subunits. Biochim Biophys Acta 142:277, 1969

8. McKenzie JM, Zaharija M: LATC in Graves' disease. Recent Prog Horm Res 33:29, 1977

9. Mori T, Kriss JP: Measurements by competitive binding radioassay of serum antimicrosomal and antithyroglobulin antibodies in Graves' disease and other thyroid disorders. J Clin Endocrinol Metab 33:688, 1971

10. Lamki L, Row VV, Volpè R: Cell-mediated immunity in Graves' disease and in Hashimoto's thyroiditis as shown by the demonstration of migration inhibition factor (MIF). J Clin Endocrinol Metab 36:358, 1973

11. Knox AJ, Von Westrap C, Row VV, et al: Demonstration of the production of thyroid stimulating immunoglobulins (HTSI) by Graves' lymphocytes cultured in vitro with phytohaemagglutinen (PHA). Metabolism 25:1217, 1976
12. Murphy BE, Potter CJ: Determination of thyroxine utilizing the property of protein binding. J Clin Endocrinol 24:187, 1964

13. Chopra IJ: A radioimmunoassay for measurement of thyroxine in unextracted serum. J Clin Endocrinol 34:938, 1972

14. Sterling K, Brenner MA: Free thyroxin in human serum: Amplified measurement with the aid of magnesium precipitation. I Clin Invest 45:153, 1966

15. Jiang $N$, Tiu KA: Determination of free thyroxin in serum by radioimmunoassay. Clin Chem 23:1679, 1977

16. Sterling K, Rafetoff $\mathrm{S}$ and Selenkow HA: T3 thyrotoxicosis. Thyrotoxicosis due to elevated serum triiodothyronine levels. JAMA 213:571, 1970

17. Ingbar SH, Braverman LE: Active form of thyroid hormone. Annu Rev Med 26:443, 1975

18. Larsen PR: Thyroidal triiodothyronine and thyroxine in Graves disease: Correlation with presurgical treatment, thyroid status and iodine content. I Clin Endocrinol Metab 41:1098, 1975

19. Irvine WJ, Toft AD: The diagnosis and treatment of thyrotoxicosis. Clin Endocrinol 5:687-707, 1976

20. Wartofsky L: Low remission after therapy for Graves' disease. JAMA 226:1083, 1973

21. Teng CS, Yeung RTT: Changes in thyroid-stimulating antibody activity in Graves' disease treated with antithyroid drug and its relationship to relapse: A prospective study. J Clin Endocrinol Metab 50:144, 1980

22. Uller RP, Van Herle AJ: Effect of therapy on serum thyroglobulin levels in patients with Graves' disease. J Clin Endocrinol Metab $46: 747,1978$

23. Shanks RG, Hadden DR, Low DC, et al: Controlled trials of propranolol in thyrotoxicosis. Lancet i:993, 1969

24. Nilsson OR, Korlberg BE, Kagedal B, et al: Non-selective and selective B-I-adrenoceptor blocking agents in the treatment of hyperthyroidism. Acta Med Scand 206:21, 1979

25. Foster RS: Morbidity and mortality after thyroidectomy. Surg Gynecol Obstet 146:423, 1978

26. Freitas JE, Swanson DP, Gross MD, et al: lodine-131: Optimal therapy for hyperthyroidism in children and adolescents? J Nucl Med 20:847, 1979

27. Dobyns BM, Sheline GE, Workman JB, et al: Malignant and benign neoplasms of the thyroid in patients treated for hyperthyroidism: A report of the cooperative thyrotoxicosis follow-up study. J Clin Endocrinol Metab 38:976, 1974

28. Pochin EE: Leukemia following radioiodine treatment of thyrotoxicosis. Br Med J 2:1545, 1960

29. Robertson JS, Gorman CA: Gonadal radioiodine treatment of thyrotoxicosis. J Nucl Med 17:826, 1976.

30. Hopwood N: Personal communication. Univ of Michigan Pediatric Graves' Disease Follow-up Study, 1980

31. Altman RP: Total thyroidectomy for the treatment of Graves' disease in children. J Pediatr Surg 8:295, 1973

32. Persik SL: Total thyroidectomy in Graves' disease in children. J Pediatr Surg 11:191, 1976

33. Roitt IM, Doniach D, Campbell PN, et al: Autoantibodies in Hashimoto's thyroiditis (lymphadenoid goiter). Lancet ii:820, 1956

34. Volpè R, Faud NR, Von Westrap C, et al: A viewpoint: The pathogenesis of Graves' disease and Hashimoto's thyroiditis. Clin Endocrinol (Oxf) 3:239, 1974

35. Allison AC: Self-tolerance and autoimmunity in the thyroid. $N$ Engl J Med 295:821, 1976

36. Hall R, Owen SG, Smart GA: Evidence for a genetic predisposition to formation of thyroid antibodies. Lancet ii:187, 1960

37. Hall R, Dingle PR, Roberts DF. Thyroid antibodies: A study of first degree relatives. Clin Genet 3:319, 1972

38. Doniach D, Roitt IM, Taylor KB: Auto immunity in pernicious anemia and thyroiditis: A family study. Ann NY Acad Sci $124: 605,1965$

39. Fisher DA, Oddie TH, Johnson DE, et al: The diagnosis of Hashimoto's thyroiditis. J Clin Endocrinol Metab 40:795, 1975

40. Amino N, Hagen SR, Yamada N, et al: Measurement of circulating thyroid microsomal antibodies by the tanned red cell hemagglutination technique: Its usefulness in the diagnosis of autoimmune thyroid diseases. Clin Endocrinol 5:115, 1976

41. Gordin A, Lamberg BA: Natural course of symptomless autoimmune thyroiditis. Lancet ii:1234, 1975 
This document is a scanned copy of a printed document. No warranty is given about the accuracy of the copy. Users should refer to the original published version of the material. 